\title{
Staphylococcus aureus Bloodstream Infections: The Association Between Age and Mortality and Functional Status
}

\author{
Preeti N. Malani, MD, ${ }^{* \neq \$ \mathcal{S}}$ Meenakshi M. Rana, MD," Mousumi Banerjee, MD, ${ }^{*}$ and \\ Suzanne F. Bradley, $M D^{* \dagger \neq \mathcal{S}}$
}

OBJECTIVES: To assess the association between Staphy-
lococcus aureus (S. aureus) blood stream infections (BSIs)
and morbidity and mortality in older adults. DESIGN: Retrospective review.

SETTING: Veterans Affairs Ann Arbor Healthcare System.

PARTICIPANTS: All patients with S. aureus BSI during 2004/05.

MEASUREMENTS: Outcomes included in-hospital and 6-month mortality, as well as need for subacute care.

RESULTS: Sixty-eight patients with $S$. aureus BSI were identified (mean age $63.5 \pm 13.0$ ). Outcomes of interest included in-hospital mortality (19.1\%), 6-month mortality $(33.8 \%)$, and need for subacute care $(65.4 \%)$. Univariate analysis identified several predictors of death, including older age, chronic renal insufficiency, catheter-related infection, Charlson weighted index of comorbidity score, and infection with methicillin-resistant $S$. aureus (MRSA). Multivariable analysis demonstrated that older age (odds ratio $(\mathrm{OR})=1.1, P<.01)$, chronic renal insufficiency $(\mathrm{OR}=$ 16.6, $P=.01)$, and MRSA infection $(\mathrm{OR}=5.1, P=.03)$ were independently associated with 6-month mortality. These results suggest that, for every decade increase in age, the odds of death within 6 months of $S$. aureus BSI doubles $(\mathrm{OR}=1.1)$. Chronic renal insufficiency was also independently associated with in-hospital mortality. Of the previously community-dwelling patients $(\mathrm{n}=50), 41$ survived hospitalization, of whom $22(53.7 \%)$ required subacute care after discharge.

CONCLUSION: Better understanding of the epidemiology of $S$. aureus BSI in older patients and validation of risk

From the *Department of Internal Medicine, Division of Infectious Diseases, ${ }^{\dagger}$ Department of Internal Medicine, Division of Geriatric Medicine, ${ }^{\ddagger}$ Ann Arbor Veterans Affairs Healthcare System, and ${ }^{\S}$ Geriatric Research Education and Clinical Center, University of Michigan Health System, Ann Arbor, Michigan; and "Department of Internal Medicine, Division of General Internal Medicine, and "Department of Biostatistics, School of Public Health, University of Michigan, Ann Arbor, Michigan.

Address correspondence to Preeti N. Malani, MD, VA Ann Arbor Healthcare System, 2215 Fuller Road, 111-I, 8th floor, Ann Arbor, MI 48105. E-mail: pmalani@umich.edu

DOI: 10.1111/j.1532-5415.2008.01823.x factors for poor functional outcomes and death should be the focus of future prospective studies. J Am Geriatr Soc 56:1485-1489, 2008.

Key words: S. aureus; aging; bloodstream infections

$\mathrm{D}$ espite vast improvements in medical care, adjusted rates of hospitalization for bloodstream infection (BSI) continue to climb in older adults. ${ }^{1,2}$ In recent years, death rates due to BSI have risen two to three times in persons aged 65 and older, seven times in those aged 75, and 20 times in those aged 85 and older. ${ }^{2}$ In some series, overall BSI-associated mortality has been reported to be greater than $50 \%$ in older adults, with the highest mortality rates associated with Staphylococcus aureus (S. aureus) infection. ${ }^{1-14}$

S. aureus BSIs are among the most morbid of common infections, and the incidence continues to increase at a higher rate than BSI caused by other organisms. ${ }^{15-18}$ The epidemiology of $S$. aureus BSI in elderly people differs from that of younger patients in that older adults are more likely to have catheter- and device-related infections and less likely to have infections related to intravenous drug use. ${ }^{3,13,18}$ Older adults also have a higher incidence of methicillin-resistant strains, reflecting the higher rates of healthcare-associated acquisition. ${ }^{3,4,18}$ Associated risk factors for development of $S$. aureus BSI include a higher incidence of comorbid illness, such as congestive heart failure, chronic pulmonary disease, hypertension, and diabetes mellitus. Risk factors for death include methicillin resistance, age, renal failure, length of mechanical ventilation, and presence of devices. ${ }^{3,15-18}$

In the general population, recent studies of $S$. aureus BSI demonstrate overall 30-day mortality rates of $30 \%$ despite appropriate antimicrobial therapy. ${ }^{16}$ Few studies specifically assess prognosis and outcomes from infection in older adults. ${ }^{19}$ Despite high (and increasing) incidence and substantial overall mortality, $S$. aureus BSI remains under- 
studied, especially in elderly people. Although mortality is higher in older adults with $S$. aureus BSI, the effect of infection on physical and cognitive functional status and long-term care needs is not known. It is likely that older adults not only suffer higher mortality from $S$. aureus BSI, but also experience longer recovery times and more-persistent functional impairment than younger adults, leading to prolonged hospitalization, need for subacute care, and higher healthcare costs.

To better understand the relationship between older age and clinical outcomes and to identify other variables of interest, a retrospective study was conducted of all community- and healthcare-associated S. aureus BSI in patients admitted to the Veterans Affairs (VA)Ann Arbor Healthcare System during 2004/05. In addition to mortality (in-hospital and 6-month), the need for subacute care was also assessed as a marker of functional status, and available Minimum Data Set (MDS) records were examined for information on activities of daily living (ADLs) and mobility. The findings are presented in this article.

\section{METHODS}

\section{Overall Research Design}

The VA Ann Arbor Healthcare System (AAVA) is a 150-bed tertiary care facility closely affiliated with the University of Michigan Health System. S. aureus BSI was defined as any blood culture positive for $S$. aureus with an appropriate clinical presentation. Baseline demographic data, clinical conditions, and characteristics of the BSI were recorded. In addition, comorbidity was measured using the Charlson weighted index of comorbidity (WIC). ${ }^{20}$ Outcomes of interest included in-hospital mortality and death within 6 months of the development of the BSI. Need for subacute care after discharge in patients who survived hospitalization was also recorded as a measure of diminished functional status. The admitting service generally determined the need for subacute care in consultation with the divisions of geriatric medicine and infectious diseases. Patients requiring subacute care were admitted to the AAVA's attached extended care center (ECC) or discharged to a community nursing center. The institutional review board of the AAVA approved this study.

\section{Data Analysis}

Univariate analysis using simple logistic regression identified risk factors for death in patients with $S$. aureus BSI. A two-tailed $P$-value less than .05 was considered statistically significant. Crude odds ratios (ORs) and 95\% confidence intervals (CIs) were calculated. Age was represented in a continuous fashion in the model with 1 year serving as the unit of measure. Variables that were significant at a $P$-value of .20 in univariate analysis and variables that had a priori clinical significance were then analyzed using multivariable logistic regression modeling. All statistical analysis was performed using SAS 9.1 (SAS Institute Inc., Cary, NC).

\section{RESULTS}

Patient characteristics are listed in Table 1 . Sixty-eight patients with a $S$. aureus BSI with a mean age of $63.5 \pm 13$ (range 20-89) were identified; all but one were male
Table 1. Characteristics of 68 Patients with Staphylococcus aureus Blood Stream Infections

\begin{tabular}{lc}
\hline \multicolumn{1}{c}{ Characteristic } & Value \\
\hline Age, mean \pm SD & $63.5 \pm 13.2$ \\
Male, $\mathrm{n}(\%)$ & $67(98.5)$ \\
Comorbidities, $\mathrm{n}(\%)$ & \\
\hline Hypertension & $48(70.6)$ \\
\hline Coronary artery disease & $29(42.6)$ \\
\hline Congestive heart failure & $15(22.1)$ \\
\hline Peripheral vascular disease & $22(32.3)$ \\
\hline Diabetes mellitus & $34(50)$ \\
\hline End-stage renal disease & $9(13.2)$ \\
\hline Chronic renal insufficiency* & $8(11.8)$ \\
\hline Pulmonary disease & $18(26.5)$ \\
\hline Cirrhosis & $10(14.7)$ \\
\hline Psychiatric illness & $15(22.1)$ \\
\hline Malignancy & $15(22.1)$ \\
\hline Stroke & $10(14.7)$ \\
\hline Recent hospitalization, $\mathrm{n}(\%)^{\dagger}$ & $22(32.3)$ \\
\hline Need for enteral feeding, $\mathrm{n}(\%)$ & $6(8.8)$ \\
\hline Charlson weighted index score, mean $\pm \mathrm{SD}$ & $5.6 \pm 2.5$ \\
\hline Source of infection, $\mathrm{n}(\%)$ & $22(32.3)$ \\
\hline Catheter & $14(20.6)$ \\
\hline Endocarditis & $18(26.5)$ \\
\hline Wound & $11(16.2)$ \\
\hline Respiratory tract & $11(16.2)$ \\
\hline Unknown source & $6(8.8)$ \\
\hline Osteoarticular & $2(3.0)$ \\
\hline Genitourinary & \\
\hline
\end{tabular}

${ }^{*}$ Baseline serum creatinine of $2 \mathrm{mg} / \mathrm{dL}$.

${ }^{\dagger}$ Hospital admission within previous 30 days.

$\mathrm{SD}=$ standard deviation.

(98.5\%). (Figure 1) Common medical comorbidities of patients were hypertension $(70.6 \%)$, diabetes mellitus $(50 \%)$, and coronary artery disease $(42.6 \%)$. The most common sources of $S$. aureus infection were catheter related $(32.2 \%)$, wound related $(26.5 \%)$, and endocarditis

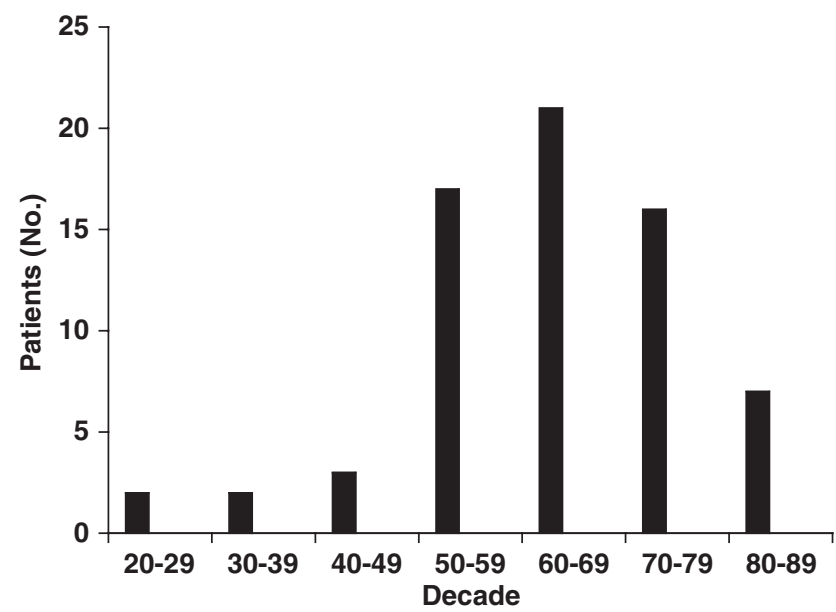

Figure 1. Age distribution of patients with Staphylococcus aureus bloodstream infection according to decade $(\mathrm{N}=68)$. 
Table 2. Univariate Analysis of the Effect of Patient Characteristics on In-Hospital and 6-Month Mortality from Staphylococcus aureus Bloodstream Infections

\begin{tabular}{|c|c|c|c|c|c|c|c|c|}
\hline $\begin{array}{l}\text { Characteristic and } \\
\text { Source of Infection }\end{array}$ & $\begin{array}{l}\text { Survived to } \\
\text { Discharge } \\
(n=55)\end{array}$ & $\begin{array}{l}\text { Died in } \\
\text { Hospital } \\
(n=13)\end{array}$ & $\begin{array}{l}\text { Unadjusted } \\
\text { OR }(95 \% \mathrm{Cl})\end{array}$ & $P$-Value & $\begin{array}{c}\text { Alive at } \\
6 \text { Months } \\
(n=45)\end{array}$ & $\begin{array}{c}\text { Dead at } \\
6 \text { Months } \\
(n=23)\end{array}$ & $\begin{array}{l}\text { Unadjusted } \\
\text { OR }(95 \% \mathrm{Cl})\end{array}$ & $P$-Value \\
\hline \multicolumn{9}{|l|}{ Patient characteristics } \\
\hline Age, mean $\pm \mathrm{SD}^{*}$ & $62.3 \pm 14$ & $68.9 \pm 8$ & $1.05(1.0-1.1)$ & .10 & $60.6 \pm 14$ & $69.4 \pm 9$ & $1.07(1.0-1.1)$ & .01 \\
\hline Charlson index, mean $\pm \mathrm{SD}$ & $5.2 \pm 2.5$ & $7.1 \pm 2.1$ & $1.38(1.0-1.8)$ & .02 & $4.9 \pm 2.2$ & $6.9 \pm 2.5$ & $1.5(1.1-1.9)$ & $<.001$ \\
\hline Chronic renal insufficiency, $\mathrm{n}(\%)$ & $4(7.2)$ & $4(30.8)$ & $5.7(1.2-27)$ & .05 & $2(4.4)$ & $6(26.1)$ & $7.5(1.4-41)$ & .02 \\
\hline End-stage renal disease, $\mathrm{n}(\%)$ & $7(12.7)$ & $2(15.4)$ & $1.3(0.2-6.8)$ & .64 & $4(8.8)$ & $5(21.7)$ & $2.8(0.7-12)$ & .25 \\
\hline Enteral feeding, $\mathrm{n}(\%)$ & $4(7.2)$ & $2(15.4)$ & $2.3(0.4-14)$ & .99 & $2(4.4)$ & $4(17.4)$ & $4.5(0.7-27)$ & .20 \\
\hline \multicolumn{9}{|l|}{ Source of infection, $\mathrm{n}(\%)$} \\
\hline Catheter related & $16(29.1)$ & $6(46.2)$ & $2.1(0.6-7.2)$ & .24 & $11(24.4)$ & $11(47.8)$ & $2.8(1.0-8.2)$ & .05 \\
\hline
\end{tabular}

* Odds ratio (OR) for age reported for 1-year unit.

$\mathrm{CI}=$ confidence interval.

(20.6\%). Thirty-six episodes of BSI $(52.9 \%)$ were secondary to MRSA, the remaining $32(47.1 \%)$ were secondary to methicillin-sensitive $S$. aureus (MSSA).

Of the 68 patients, 50 lived in the community before admission, either alone $(\mathrm{n}=11)$ or with family members $(\mathrm{n}=39)$. Another 16 patients were admitted from an extended care setting, either chronic or subacute care. The remaining two patients were transferred from another hospital, although both lived in the community before admission to the outside hospital.

Thirteen of the 68 patients (19\%) died before discharge during the initial hospitalization. An additional 10 patients died within 6 months of developing the S. aureus BSI (Table 2). Of the 55 patients who survived hospitalization, 36 $(65.4 \%)$ required admission to an extended care facility. Twenty-six of the 36 extended care patients received subacute care at the VA's ECC. Primary reasons for subacute care included impaired mobility and nutrition, wound care, and need for parenteral antimicrobial therapy. The ECC does not offer long-term care. The AAVA has a well-established home antimicrobial program, ${ }^{21}$ so few patients were sent to subacute care just to complete a course of therapy. Of the previously community-dwelling patients $(\mathrm{n}=50), 41$ survived hospitalization, of whom $22(53.7 \%)$ required subacute care after discharge. Not surprisingly, older age predicted need for subacute care in previously independent patients $(\mathrm{OR}=1.05, P=.04)$.

MDS data were available for 26 of the $68(38 \%)$ patients. Nine of the 26 were independent or needed minimal assistance with ADLs and mobility. The remaining 17 $(65.4 \%)$ patients required a minimum of assistance with two or more ADLs, bed mobility, or both from one person. Of the $17,12(70.5 \%)$ were initially admitted from the community, all living independently alone or with family. The other five patients were admitted from a subacute or skilled care setting.
Univariate analysis identified several factors associated with mortality (in-hospital and 6-month) including age, chronic renal insufficiency (defined as serum creatinine $\geq 2 \mathrm{mg} / \mathrm{dL}$ ), catheter-related infection, Charlson WIC score, and MRSA infection (Table 2). Of these characteristics, three remained independently associated with 6-month mortality based on multivariate analysis: age $(\mathrm{OR}=1.1$, $P<.01)$, chronic renal insufficiency $(\mathrm{OR}=16.6, P=.01)$, and MRSA infection $(\mathrm{OR}=5.1, P=.03)$ (Table 3). The adjusted OR for age suggests that, for every 10 years increase in age, the odds of dying within 6 months of $S$. aureus BSI doubles. Chronic renal insufficiency was also independently associated with in-hospital mortality $(\mathrm{OR}=7.0$, $P=.03)$; age demonstrated borderline significance for inhospital mortality $(\mathrm{OR}=1.05, P=.08)$.

\section{DISCUSSION}

The preceding results offer further evidence that older age is an independent predictor of mortality associated with $S$. aureus BSI. These results suggest that, for every 10 years increase in age, the odds of dying within 6 months of $S$. aureus $\mathrm{BSI}$ doubles $(\mathrm{OR}=1.1)$. In addition to significant mortality, S. aureus BSI is perhaps associated with a decline in functional status in older adults. Specifically, the need for subacute care after discharge was seen in two-thirds of patients who survived hospitalization. Even in those who lived independently in the community before admission, more than half required care after discharge. Age predicted the need for such care in previously independent patients $(\mathrm{OR}=1.05, P=.04)$, as well as in the overall study population. Although there are multiple reasons why patients require subacute care posthospitalization, including antimicrobial administration and wound care, the limited MDS records available suggested significant impairments in mo- 
Table 3. Multivariate Analysis of the Association of Select Patient Characteristics on In-Hospital and 6-Month Mortality from Staphylococcus aureus Bloodstream Infections

\begin{tabular}{|c|c|c|c|c|c|c|}
\hline \multirow[b]{3}{*}{ Characteristic } & \multicolumn{3}{|c|}{ In-Hospital Mortality } & \multicolumn{3}{|c|}{ 6-Month Mortality } \\
\hline & Unadjusted & Adjusted* & \multirow[b]{2}{*}{$P$-Value } & Unadjusted & Adjusted* & \multirow[b]{2}{*}{$P$-Value } \\
\hline & \multicolumn{2}{|c|}{ OR $(95 \% \mathrm{Cl})$} & & \multicolumn{2}{|c|}{ OR (95\% Cl) } & \\
\hline $\mathrm{Age}^{\dagger}$ & $1.05(1.0-1.1)$ & $1.05(1.0-1.1)$ & .08 & $1.07(1.0-1.1)$ & $1.1(1.0-1.2)$ & $<.01$ \\
\hline Chronic renal insufficiency & $5.7(1.2-27)$ & $7.0(1.2-40.8)$ & .03 & $7.5(1.4-41)$ & $16.6(1.8-153)$ & .01 \\
\hline Catheter-related infection & $2.1(0.6-7.2)$ & $4.1(8-22.3)$ & .10 & $2.8(1.0-8.2)$ & $4.5(0.7-27)$ & .10 \\
\hline MRSA & $2.3(0.6-8.5)$ & $1.8(0.4-8.0)$ & .46 & $3.8(1.3-11.7)$ & $5.1(1.1-22.9)$ & .03 \\
\hline
\end{tabular}

* Odds ratios (ORs) and $95 \%$ confidence intervals (CIs) are based on a multivariable logistic regression adjusting for age, chronic renal insufficiency, end-stage renal disease, recent hospitalization, catheter-related infection, endocarditis, Charlson weighted comorbidity index, and methicillin-resistant Staphylococcus aureus (MRSA).

${ }^{\dagger}$ OR for age reported for 1-year unit.

bility and cognitive function in patients who previously lived independently.

Before planned prospective studies related to functional status and $S$. aureus infection were begun, this pilot study was performed to identify potential predictors of mortality. Although its retrospective design, a small sample size, and incomplete functional assessments limited the present study, the results suggest an association between age and functional decline and between age and mortality. This study was conducted at a VA hospital, which serves a population that tends to be overwhelmingly male and relatively young, with high rates of comorbid illnesses. Although $98 \%$ of the study patients were male, these preliminary results are generalizable, given that there is no evidence in the literature that outcome for $S$. aureus BSI varies according to sex.

The difference between in-hospital and 6-month mortality and the possible effect of age is intriguing. The results suggest that, even if an older adult survives hospitalization for a $S$. aureus BSI, a significant risk of debility at discharge and death at 6 months remains. This potential difference offers further support for future studies to assess functional status and the effect of rehabilitation on outcomes from $S$. aureus BSI. Better understanding of the epidemiology of $S$. aureus BSI in older patients, as well as validation of risk factors for poor functional outcomes and death, can also help direct future studies of focused interventions such as decolonization, antimicrobial-coated devices, and even vaccines. $^{22-24}$

Assessing who among elderly people develops $S$. aureus BSI, who is likely to survive, and what effect is seen on functional status will be important in developing prognostic indices and new treatment algorithms. ${ }^{19,25}$

\section{ACKNOWLEDGMENTS}

This work was supported in part by the AAVA; the Veterans Education Research Association of Michigan; the Geriatric Research, Education and Clinical Center, the VA Merit Review Program, the John A. Hartford Foundation Center of Excellence; and the University of Michigan K30 Training Program in Clinical Research. It was presented in part at the 17th Annual Scientific Meeting of the Society for Health- care Epidemiology of America, Baltimore, Maryland, April 14-17, 2007 (Poster 92).

Conflict of Interest: Malani, Rana, Banerjee, and Bradley report no conflicts of interest related to this work.

Author Contributions: Malani: study design, data collection, analysis and interpretation of data, manuscript preparation. Rana: data collection. Banerjee: analysis and interpretation of data. Bradley: study design, interpretation of data, manuscript preparation.

Sponsor's role: None.

\section{REFERENCES}

1. Pinner RW, Teutsch SM, Simonsen L et al. Trends in infectious diseases mortality in the United States. JAMA 1996;275:189-193.

2. McBean M, Rajamani S. Increasing rates of hospitalization due to septicemia in the US elderly population, 1986-1997. J Infect Dis 2001;183:596-603.

3. McClelland RS, Fowler VG, Sanders LL. Staphylococcus aureus bacteremia among elderly vs. younger adult patients: Comparison of clinical features and mortality. Arch Intern Med 1999;159:1244-1247.

4. Bader MS. Staphylococcus aureus bacteremia in older adults: Predictors of 7 day mortality and infection with a methicillin-resistant strain. Infect Control Hosp Epidemiol 2006;27:1219-1225.

5. Tacconelli E, Pop-Vicas AE, D’Agata EMC. Increased mortality among elderly patients with methicillin-resistant Staphylococcus aureus bacteremia. J Hosp Infect 2006;64:251-256.

6. Deulofeu F, Cervello B, Capell S et al. Predictors of mortality in patients with bacteremia: The importance of functional status. J Am Geriatr Soc 1998;46:14-18.

7. Gavazzi G, Mallaret MR, Couturier P et al. Bloodstream infection: Difference between young-old, old and old-old patients. J Am Geriatr Soc 2002;50:16671673.

8. Meyers BR, Sherman E, Mendelson MH et al. Bloodstream infections in the elderly. Am J Med 1989;86:379-384.

9. Esposito AL, Gleckman RA, Cram S et al. Community-acquired bacteremia in the elderly: Analysis of one hundred consecutive episodes. J Am Geriatr Soc 1980;28:315-319

10. Leibovici L, Samra Z, Koingsberger $\mathrm{H}$ et al. Long-term survival following bacteremia or fungemia. JAMA 1995;274:807-812

11. Ackermann RJ, Monroe PW. Bacteremic urinary tract infection in older people. J Am Geriatr Soc 1996;44:927-933.

12. Leibovici L, Pitlik SD, Konisberger $\mathrm{H}$ et al. Bloodstream infection in patients older than eighty years. Age Ageing 1993;22:431-442.

13. Lee CC, Chen SY, Chang IJ et al. Comparison of clinical manifestations and outcome of community-acquired bloodstream infections among the oldest old, elderly, and adult patients. Medicine (Baltimore) 2007;86:138-144.

14. Simor AE, Ofner-Agostini M, Paton S et al. Clinical and epidemiologic features of methicillin-resistant Staphylococcus aureus in elderly hospitalized patients. Infect Control Hosp Epidemiol 2005;26:838-841.

15. Cosgrove SE, Sakoulas G, Perencevich EN et al. Comparison of mortality associated with methicillin-resistant and methicillin susceptible Staphylococcus aureus bacteremia: A meta-analysis. Clin Infect Dis 2003;36:53-59. 
16. Chang FY, MacDonald BB, Peacock JE et al. A prospective multicenter study of Staphylococcus aureus bacteremia: Incidence of endocarditis, risk factors for mortality, and clinical impact of methicillin resistance. Medicine (Baltimore) 2003;82:322-332.

17. Blot SI, Vanderwoude KH, Hoste EA et al. Outcome and attributable mortality in critically ill patients with bacteremia involving methicillin-suceptible and methicillin-resistant Staphylococcus aureus. Arch Intern Med 2002;162:2229-2235.

18. Bradley SF. Staphylococcus aureus infections and antibiotic resistance in older adults. Clin Infect Dis 2002;34:211-216.

19. High KP, Bradley S, Loeb M et al. A new paradigm for clinical investigation of infectious syndromes in older adults: Assessment of functional status as a risk factor and outcome measure. Clin Infect Dis 2005;40:114-122.

20. Charlson ME, Pompei P, Ales KL et al. A new method for classifying prognostic comorbidity in longitudinal studies: Development and validation. J Chronic Dis 1987;40:373-383.
21. Cox A, Malani PN, Wiseman SW et al. Home intravenous antimicrobial infusion therapy: A viable option in older adults. J Am Geriatr Soc 2007;55 $645-650$.

22. Mody L, Kauffman CA, McNeil SA et al. Mupirocin decolonization of Staphylococcus aureus carriers in two long-term care facilities: A randomized, double-blinded, placebo-controlled trial. Clin Infect Dis 2003;37: 1467-1474.

23. Veenstra DL, Saint S, Sullivan SD. Cost-effectiveness of antiseptic-impregnated central venous catheters for the prevention of catheter-related blood stream infection. JAMA 1999;282:554-560.

24. Shinefield H, Black S, Fattom A et al. Use of a Staphylococcus aureus conjugate vaccine in patients receiving hemodialysis. N Engl J Med 2002;346:491-496.

25. Juthani-Mehta M, Quagliarello VJ. Prognostic scoring systems for infectious diseases: Their applicability to the care of older adults. Clin Infect Dis 2004; 38:692-696. 